\title{
Isolated Antiphospholipid Syndrome of the Central Nervous System With Positive Lupus Anticoagulant: Two Case Reports
}

\author{
Kazuya Takahashi
}

\begin{abstract}
I present two cases of isolated antiphospholipid syndrome (APS) of the central nervous system (CNS) with positive lupus anticoagulant (LA). Both these cases were of the remitting-relapsing type, and optic neuritis and transverse myelitis were not observed. Anticardiolipin antibodies (aCL), anti- $\beta_{2}$ glycoprotein-I antibodies, and anti-aquaporin- 4 antibodies were not detected. Previous reports suggested that both multiple sclerosis (MS) patients with a positive aCL titer and APS patients with an MS-like disease frequently exhibit a neuromyelitis optica-like clinical course and they were positive for aCL but not LA. These results indicate that LA-positive APS and aCL-positive APS cases might differ in terms of CNS involvement.
\end{abstract}

Keywords: Antiphospholipid syndrome; Multiple sclerosis; Lupus anticoagulant; Kaolin clotting time; Anticardiolipin antibodies

\section{Introduction}

Antiphospholipid syndrome (APS) and multiple sclerosis (MS) are both representative autoimmune diseases; however, the management of these two diseases is completely different. Interferon, a first-line drug in the management of MS, induces systemic lupus erythematosus (SLE), a major underlying disease in the case of APS [1, 2]. Although the only neurological manifestation of APS according to the recent published Sydney criteria is the occurrence of ischemic

Manuscript accepted for publication November 11, 2010

Department of Neurology,National Hospital Organization Iou National Hospital, Ni73-1, Iwade-machi, Kanazawa 920-0192, Japan. Email: ktakaha@ioudom.hosp.go.jp

doi: $10.4021 / \mathrm{jmc} 81 \mathrm{w}$ neurological complications, other non-ischemic neurological complications such as demyelination associated with MS are also observed in patients with APS [3-5]. Here, I report 2 cases of isolated antiphospholipid syndrome of the central nervous system with positive lupus anticoagulant (LA).

\section{Case Report}

\section{Case 1}

A 29-year-old woman was admitted to our hospital for treatment of left facial palsy and vertigo. The findings of a general examination performed on admission were normal. On neurological examination, she was found to have decreased sensation on the left side of her face, left peripheral facial palsy, and right rotatory nystagmus. Serological examination revealed the presence of antithyroglobulin antibodies $(1: 1600$, normal $<1: 100)$, antimicrosomal antibodies $(1: 400$, normal $<1: 100)$ and a low titer of anticardiolipin antibodies $(\mathrm{aCL})(19.7 \mathrm{U} / \mathrm{mL}$, normal $<10 \mathrm{U} / \mathrm{mL})$. Tests for anti$\beta_{2}$ glycoprotein-I antibodies $\left(\beta_{2} \mathrm{GPI}\right)$, antinuclear antibodies (ANA), and anti-aquaporin-4 antibodies (AQP4) were negative. Lupus anticoagulant (LA) sampling after steroid treatment was negative. The cerebrospinal fluid was clear with a normal cell count and $\operatorname{IgG}$ index $(0.46$, normal $<0.70)$, but the protein level was slightly elevated $(46 \mathrm{mg} / \mathrm{dL}$, normal $<40 \mathrm{mg} / \mathrm{dL}$ ). Oligoclonal bands (OB) were not observed. Magnetic resonance imaging (MRI) with gadolinium enhancement revealed a lesion in the left middle peduncle (Fig. 1A). She was intravenously administered methylprednisolone. After treatment, her symptoms improved gradually. Interferon treatment to prevent disease progression was not provided because she intended to get pregnant. She dropped out of our regular experiment several months after discharge. At the age of 34 years, she visited our hospital for a reexamination. Except for a slightly enlarged right cervical lymph node, the general examination findings were normal. Neurological examination revealed mild right hemiparesis, hypoesthesia in the right upper and lower limb, and hypotonic neurogenic bladder. Blood test results were not significant except for a high titer of antithyroid antibodies. LA was 


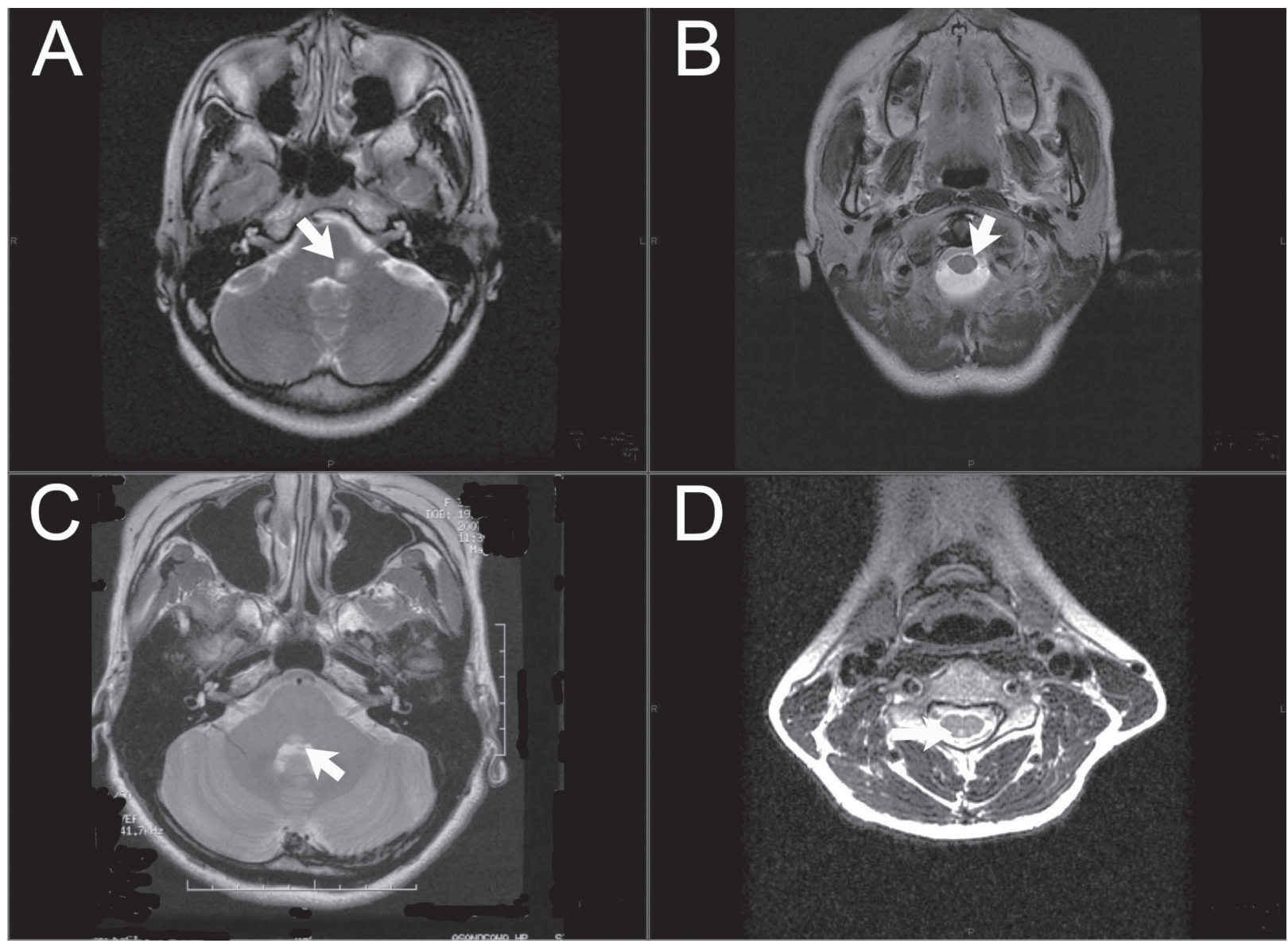

Figure 1. Magnetic resonance imaging (MRI) scans. (A, B) MRI scans of case 1: T2-weighted images show hyperintense areas in the left middle peduncle (A) and in the medulla (B); (C, D) MRI scans of case 2: T2-weighted images show hyperintense areas in the midbrain $(C)$ and in the right posterior funiculus (D).

detected with the kaolin clotting time (KCT) assay, but not with the dilute Russell viper venom time (dRVVT) assay. $\mathrm{aCL}$ and $\beta_{2}$ GPI were not detected, and the activated partial thromboplastin time (APTT) was normal. MRI revealed the previously detected lesion in the left middle peduncle, and a new lesion was detected on the left side of the medulla (Fig. 1B). Periventricular, juxtacortical, and spinal cord lesions, which are indicative of MS, were not detected on the MRI scan. She had not experienced systemic symptoms such as arthralgia, and had not received any medication regularly. The presence of LA in her plasma was confirmed repeatedly. She received continuous treatment with low doses of aspirin but not interferon. Since then, she has not suffered a relapse for 2 years.

\section{Case 2}

A 35-year-old woman visited the neurology department of a general hospital for the treatment for double vision. She was diagnosed with right medial longitudinal fasciculus (MLF) syndrome, and MRI with gadolinium enhancement revealed a T2 high-intensity lesion in the midbrain (Fig. 1C). She received oral steroid treatment and her symptoms improved immediately. She became symptom free and did not receive interferon treatment for prevention of an MS relapse. One year later, a follow-up MRI was conducted. Although there were no clinical signs noted, a new lesion had developed in the left internal capsule. At the age of 37 years, she visited our hospital because she experienced a painful numbness in her right arm. With regard to the neurological symptoms, she experienced painful hyperesthesia in an area corresponding to the right C5 segment, and the MRI revealed a T2 highintensity lesion in the left posterior funiculus extending from segment C4 to C5 (Fig. 1D). No periventricular and juxtacortical lesions were detected on the brain MRI scan. The $\mathrm{KCT}$ assay revealed the presence of LA in the serum, but LA was not detected with the dRVVT assay. ANA, aCL, $\beta_{2}$ GPI, and AQP4 were absent. She received intravenous methyl- 
prednisolone therapy, and her symptoms improved. She gave birth to a child and did not suffer a miscarriage. She never experienced systemic symptoms such as arthralgia, and did not receive any medication regularly. After repeated confirmation of the presence of LA in her plasma, she received continuous treatment with low doses of aspirin. Since then, she has not suffered a relapse for 2.5 years.

\section{Discussion}

Previous studies have revealed that $8 \%$ of patients with APS are also diagnosed with MS or MS-like disease. Further, these patients frequently present with transverse myelitis or optic neuritis [6]. On the other hand, Fukazawa et al reported two MS patients in Japan who had a positive aCL titer and presented with optic neuritis and transverse myelitis: there were probably cases of neuromyelitis optica (NMO) [7]. In addition, it has been reported that patients with NMO spectrum disorder along with SLE and APS often have positive AQP4 titers [8]. Ferreira et al reported that CNS involvement in APS is indicated by the predominance of subcortical lesions in the brain and long spinal lesions [9]. Most cases with CNS involvement had positive aCL titers, but they were negative for LA [9]. These reports suggest that MS patients with a positive aCL titer and APS patients with MS-like disease frequently exhibit an NMO-like clinical course and positive test for aCL but not LA. However, the two patients described in this report did not have optic neuritis or transverse myelitis, and their tests were negative for AQP4, aCL, and $\beta_{2}$ GPI but positive for LA. These results indicate that LA-positive APS and aCL-positive APS cases might differ in terms of CNS involvement.

\section{Conflicts of Interest}

The author has no financial disclosures to declare and no conflicts of interest to report. There is no fund for this study.

\section{Acknowledgments}

The author thanks Dr. Hidesaku Asakura of the Department of Hematology of the University of Kanazawa for measuring
LA in the serum and Prof. Keiko Tanaka of the Department of Neurology of Kanazawa Medical University for measuring AQP4 in the serum.

\section{References}

1. Bonaci-Nikolic B, Jeremic I, Andrejevic S, Sefik-Bukilica M, Stojsavljevic N, Drulovic J. Anti-double stranded DNA and lupus syndrome induced by interferon-beta therapy in a patient with multiple sclerosis. Lupus 2009;18(1):78-80.

2. Crispin JC, Diaz-Jouanen E. Systemic lupus erythematosus induced by therapy with interferon-beta in a patient with multiple sclerosis. Lupus 2005;14(6):495-496.

3. Miyakis S, Lockshin MD, Atsumi T, Branch DW, Brey RL, Cervera R, Derksen RH, et al. International consensus statement on an update of the classification criteria for definite antiphospholipid syndrome (APS). J Thromb Haemost 2006;4(2):295-306.

4. Miesbach W. Neurologic symptoms as a feature of the antiphospholipid syndrome. Semin Thromb Hemost 2008;34(3):286-289.

5. Chapman J, Rand JH, Brey RL, Levine SR, Blatt I, Khamashta MA, Shoenfeld Y. Non-stroke neurological syndromes associated with antiphospholipid antibodies: evaluation of clinical and experimental studies. Lupus 2003;12(7):514-517.

6. JW IJ, Conti-Kelly AM, Greco P, Abedi M, Amos M, Provenzale JM, Greco TP. Anti-phospholipid antibodies in patients with multiple sclerosis and MS-like illnesses: MS or APS? Lupus 1999;8(2):109-115.

7. Fukazawa T, Moriwaka F, Mukai M, Hamada T, Koike T, Tashiro K. Anticardiolipin antibodies in Japanese patients with multiple sclerosis. Acta Neurol Scand 1993;88(3):184-189.

8. Mehta LR, Samuelsson MK, Kleiner AK, Goodman AD, Anolik JH, Looney RJ, Schwid SR. Neuromyelitis optica spectrum disorder in a patient with systemic lupus erythematosus and anti-phospholipid antibody syndrome. Mult Scler 2008;14(3):425-427.

9. Ferreira S, D'Cruz DP, Hughes GR. Multiple sclerosis, neuropsychiatric lupus and antiphospholipid syndrome: where do we stand? Rheumatology (Oxford) 2005;44(4):434-442. 